\title{
Ultrasound localization microscopy and functional ultrasound reveal atypical features of the live trigeminal ganglion
}

\section{Annabelle Réaux-Le Goazigo}

Inserm

\section{Benoit Beliard}

Inserm, ESPCI Paris, CNRS, PSL Research 邓University

Line Rahal

Inserm, ESPCI Paris, CNRS, PSL Research 『University

Julien Claron

Inserm, ESPCI Paris, CNRS, PSL Research 『University

Noémi Renaudin

Inserm, ESPCI Paris, CNRS, PSL Research 囚University

\section{Isabelle Rivals}

ESPCI Paris, PSL Research खUniversity, UMRS 1158

\section{Miguel Thibault}

Inserm, ESPCI Paris, CNRS, PSL Research 囚University

\section{Mohamed Nouhoum}

Iconeus

Thomas Deffieux

Inserm

Mickael Tanter

Inserm

Sophie PEZET ( $\square$ sophie.pezet@espci.fr)

Inserm, ESPCI Paris, CNRS, PSL Research囚University https://orcid.org/0000-0002-3305-3315

\section{Article}

Keywords: Neuroimaging, neurovascular coupling, cornea, blood flow, super-resolution, ultrasound localization microscopy, neuroimaging

Posted Date: May 24th, 2021

DOl: https://doi.org/10.21203/rs.3.rs-535971/v1 
License: (c) (i) This work is licensed under a Creative Commons Attribution 4.0 International License. Read Full License

Version of Record: A version of this preprint was published at Communications Biology on April 7th, 2022. See the published version at https://doi.org/10.1038/s42003-022-03273-4. 


\section{Ultrasound localization microscopy and functional ultrasound reveal atypical features of the live trigeminal ganglion}

Annabelle Réaux-Le-Goazigo ${ }^{1}$, Benoit Beliard ${ }^{2}$, Line Rahal ${ }^{2}$, Julien Claron ${ }^{2}$, Noémi Renaudin $^{2}$, Isabelle Rivals ${ }^{3}$, Miguel Thibaut ${ }^{2}$, Mohamed Nouhoum ${ }^{2,4}$, Thomas Deffieux ${ }^{2}$, Mickael Tanter ${ }^{2}$ and Sophie Pezet $^{2 *}$

${ }^{1}$ Sorbonne Université, INSERM U968, CNRS, Institut de la vision, 17 rue Moreau, 75012 Paris, France.

2 Physics for Medicine Paris, Inserm, ESPCI Paris, CNRS, PSL Research University - Paris, France.

${ }^{3}$ Equipe de Statistique Appliquée, ESPCI Paris, PSL Research University, UMRS 1158, 10 rue Vauquelin, 75005 Paris, France.

${ }^{4}$ Iconeus, 27 Rue du Faubourg Saint-Jacques, 75014 Paris, France

${ }^{*}$ Corresponding author: Sophie PEZET

Address: Institute of Physics for Medicine Paris, ESPCI Paris, 17 rue Moreau, 75012, Paris, FRANCE

Email: sophie.pezet@espci.fr

Number of text pages: 28

Number of figures: 3

Number of tables: 0

Number of supplementary materials: 4 (2 figures +2 videos)

\section{Keywords:}

Neuroimaging, neurovascular coupling, cornea, blood flow, super-resolution, ultrasound localization microscopy, neuroimaging.

\section{Short title:}

Functional imaging of the rat's trigeminal ganglion 


\section{ABSTRACT}

The functional imaging of the neurovascular coupling within the trigeminal ganglion (TG) is highly challenging due to its small size and its deep localization. This study combined a methodological framework able to dive into the rat trigeminal nociceptive system by jointly providing first imaging of the trigeminal ganglion blood vasculature at microscopic resolution and the measurement of its neurovascular coupling in the rat TG evoked by corneal stimulations, a robust and clinically-relevant model. Using functional ultrasound imaging (fUS), we were able to image and quantify a strong hemodynamic response in the ipsilateral TG from anesthetized rats, evoked by mechanical or chemical stimulations of corneal nociceptive fibers to intact cornea, even though TG involves less than 300 sensory neurons. The in vivo quantitative imaging of the TG's vasculature using ultrasound localization microscopy (ULM) combined with ex-vivo (DiI) staining reveals particular features of the vascularization of the area containing the sensory neurons, that is likely the origin of this strong vaso-trigeminal response and due to the nature of this structure at the interface between the peripheral and central nervous systems. This innovative imaging approach opens the path for future studies on the mechanisms underlying changes in trigeminal local blood flow and neurovascular coupling, key mechanisms and readouts for the understanding and treatment of debilitating trigeminal pain conditions. 


\section{INTRODUCTION}

The trigeminal ganglion (TG) contains the cell body of the primary sensory neurons from the ophthalmic (V1), the maxillary (V2), and the mandibular (V3) nerves. These sensory neurons are highly specialized, as they detect and respond to a variety of chemical, mechanical and thermal stimuli applied on these branches.

Because the TG is relatively small and localized in Merkel's trigeminal cave in both human and rodents, only a limited number of studies were able to perform functional neuroimaging studies. While Bererra's and Borsook's teams published seminal works on the existence of a neurovascular coupling in the human TG (1-4), only one preclinical contrast MRI study imaged macrophage infiltration in the mouse TG using ultrasmall superparamagnetic iron oxide nanoparticle contrast in a model of alkani burn cornea (5). But, to the best of our knowledge, dynamic functional imaging of the TG was never performed in rodents due to the difficulties to access the TG. Preclinical studies investigating the physiological activity within the TG of rodents are classically based on electrophysiological recordings of single and/or

clusters of neurons $(6,7)$, as well as immunohistochemical staining using indirect markers of neuronal activation (see for review (8)). Despite the cellular resolution of these surrogates, this approach lacks the ability to follow the dynamics of these neuronal changes. Recently, the visualization of trigeminal sensory neuron activities in response to orofacial stimuli was reported ex-vivo using either voltage sensitive dye approach in decerebrated animals (9) or calcium imaging in GCaMP6 mouse line $(10,11)$. However, these experimental paradigms used were highly invasive requiring the decerebration of the animal, and therefore the disconnection between TG and the CNS. 
Functional ultrasound imaging (fUS) imaging is a relatively new versatile neuroimaging modality that allows imaging and measurement of cerebral blood volume in both human $(12,13)$, non-human primates (14) and rodents (15-19) with excellent spatial (100 to $300 \mu \mathrm{m}$ ) and temporal resolutions (down to $20 \mathrm{~ms}$ ). One of its biggest characteristics is its high sensitivity compared to fMRI (20). Indeed, during a task, the locally increased neuronal activity due to the neurovascular coupling leads to a hemodynamic response (21). In the past, fUS imaging showed to be sensitive enough to measure the cortical hemodynamic changes induced by sensory (18), olfactory (22), visual (23) stimuli in anesthetized animals, as well as auditory (24), motor tasks (14-16) in awake animals. Interestingly functional ultrasound imaging can be coupled on the same device with another emerging modality, Ultrasound Localization Microscopy (ULM), able to provide the brain vascular anatomy and hemodynamic quantification up to microscopic resolution both in rodents (25) and humans (26).

The corneal trigeminal system is particularly interesting as the cornea is the most densely innervated tissue in the body (8) whose nerve terminals are directly accessible for stimulation. Moreover, the cornea is exclusively innervated by unmyelinated C-and thinly myelinated A-delta fibers, including mechano-nociceptors that are triggered by noxious mechanical stimulation, polymodal nociceptors that are excited by mechanical, chemical, and thermal stimuli, and cold thermoreceptors that are activated by cooling $(8,27,28)$. Taking advantage of the high sensitivity of fUS imaging and the corneal nociceptive model, this study has several main objectives: first to image the TG in rat, second measure the velocity of blood flow in the TG using ULM and third to detect and measure functional activations in the ophthalmic division following corneal stimulations. We provide the first proof of concept of imaging the rat's TG, with a detection of local blood flow at a microscopic scale, and of the measurement of the hemodynamic responses evoked by the 
activation of corneal nociceptors in anesthetized animals. Our results bring forward an innovative approach to study the TG's neurovascular coupling, a key mechanism for deciphering the mechanisms of trigeminal sensitization and concomitant pain associated with trigeminal pathologies.

\section{RESULTS}

\section{Localization / imaging of the rat trigeminal ganglia using ultrafast Doppler imaging}

Taking into account that TG is a deep structure, we imaged much deeper under the brain as compared to previous studies in anaesthetized rodents $(18,19)$. Despite the signal attenuation due to the depth of imaging $(15 \mathrm{~mm})$, two-dimension scans (2D) revealed two bilateral longitudinal structures detected between the antero-posterior coordinates Bregma -3.3 and Bregma $-5.0 \mathrm{~mm}$ (Figure 1A, B, F). In addition to two-dimension scans (Figure 1A), three-dimension scans (3D, Figure 1B, Supplementary video 1) precisely localized the structure respectively to the location of the brain's vasculature.

Comparison with the tractography atlas of the Waxholm atlas (https://scalablebrainatlas.incf.org/rat/PLCJB14, $(29,30)$ ) confirmed that these bilateral structures are located under the brain at the same anteroposterior coordinates and laterality than the TG they described using tractography of peripheral fibers (Figure $1 \mathrm{C}$, Supplementary figure 1).

Considering that the doppler signal of the TG was visible at this depth despite the signal attenuation, suggests that TG is a richly vascularized structure. This was confirmed by comparison between the doppler signal in the TG and in the internal carotid (Figure 1A). We found that the blood volume measured in the TG (without correction for the attenuation) represents $44.0 \% \pm 1.4 \%$ of that of the internal carotid. 
Next, ex vivo staining of the TG vessels revealed a high density of DiI stained vessels which exhibited a tortuous morphology. Altogether, the ex vivo staining and in vivo fUS imaging data, support the idea that TG receive an important blood supply.

\section{Corneal nociceptor stimulations induce functional hyperhaemia in the ipsilateral}

\section{trigeminal ganglion}

Our anatomical study confirmed a high density of blood vessels stained in vivo using DiI in close interaction with small and medium diameter CGRP+ primary sensory neurons in the ophthalmic branch of the TG (Figure 2B,C). This anatomical organization of trigeminal CGRP nociceptors is suspected to play a role in orchestrating the hemodynamic response to corneal stimulation. Due to both the rich vascularization of the TG and the high sensitivity of fUS imaging to slight doppler changes (14), at the pixel level $(20,24)$, we postulated that fUS imaging could provide the first demonstration of hemodynamic responses in the ophthalmic division of the TG evoked by corneal stimulations. To this end, we used stimulations of the cornea as a system model, as the cornea is richly innervated by $\mathrm{C}$ and A delta fibers (Figure $2 \mathrm{~A}$ ) and has the advantage to be easily activated by external mechanical and chemical stimulations.

Despite the small number of corneal neurons (31), repetitive mechanical stimulations of the cornea induced strong and reproducible hemodynamic responses in the ipsilateral TG (Figure 2E-G, red line, $7.3 \%$ increase, $p=3.310^{-22}$ ), but not in the contralateral TG (blue line, $0.02 \%$ increase, $p=0.92$ ), the difference between the ipsilateral and contralateral delta blood volumes during stimulation being also highly significant $2.5 \%$ increase, Figure $2 \mathrm{H}, \mathrm{p}=1.710^{-23}$ ). Supplementary Video 2 shows a typical example of experiment with a visualization in real time of changes in BV during repeated mechanical corneal stimulations. Furthermore, the significant hemodynamic response remained stable 
during the duration of every single corneal stimulation, suggesting a lack of desensitization in both the peripheral C-fiber activation and neurovascular coupling.

Finally, the activation of TRPV1 polymodal corneal nociceptors using the TRPV1 ligand Capsaicin $(10 \mu \mathrm{M})$ induced a phasic and robust increased blood volume (18.9\% increase, $\mathrm{p}=9.310^{-3}$ ) in the ipsilateral TG as compared to the contralateral TG (Figure 2B-F). The BV increase lasted during all the time of exposure to the drug (1 min), but was still observed afterwards (Figure 2E).

\section{Ultrasound Localization Microscopy (ULM) imaging reveals vascular features of the trigeminal blood flow}

To assess novel information regarding the vascular characteristics of the blood supply within the TG (direction of the flow and its velocity), we used ULM implemented on the same ultrafast ultrasound scanner. This method allowed the measurement of flow of microbubbles inside blood vessels, at a microscopic scale in anesthetized animals $(25,32)$. We were able to provide a thin definition of blood vessel and the measure and direction of the blood flow in 2D. Our data confirmed i) that the TG is highly vascularized, with a dense network of tortuous blood vessels. ii) The organization of vessels does not look like any vascularized cerebral structures in the sagittal plane imaged (Figure 3C-E versus Figure 3B). Interestingly, the direction of blood flow within the TG was irregular (Figures 3F-H), with two large tendencies: a ventro-posterior flux for the caudal part (right in Figures $3 \mathrm{~F}-\mathrm{H}$ ) and the opposite on the rostral part. (left, Figure $3 \mathrm{H}$ ). Another difference with other cerebral structures, was the heterogeneity of speed in each portion of the TG, with a gradient of speed raging from 9 to $20 \mathrm{~mm} / \mathrm{sec}$ (at the top, versus in the ventral aspect of the structure, respectively). Interestingly, these velocities were as high as those measured in the local major blood vessels depicted: $a, b$, and c (Figures 3C-E, versus velocity in the TG in Figures 3F-G ). Due to their large diameter (a: $24-30 \mu \mathrm{m}$, b: $22-24 \mu \mathrm{m}$, 
c: $24-31 \mu \mathrm{m}$ ), high velocity (a: $8 \mathrm{~mm} / \mathrm{sec}, \mathrm{b}: 19 \mathrm{~mm} / \mathrm{sec}, \mathrm{c:} 10 \mathrm{~mm} / \mathrm{sec}$ ) ) and flow direction, we hypothesize that a and $\mathrm{c}$ are veins running in the ventro-anterior direction, while $\mathrm{b}$ is an artery (33), running in the opposite direction (Figure 3C-E).

\section{DISCUSSION}

Functional imaging studies of the TG in animal models are crucially needed to understand debilitating trigeminal pathologies (such as migraine, corneal pain or trigeminal neuralgia), which prevalence increases since decades and remain a therapeutic challenge (34).

However, such investigations are technically difficult to perform due to small size of the TG and its deep location. Here, we provide the first proof of concept of in-depth imaging of the TG's subtle vascularity vector fields and speed of flow in naïve anesthetized rats. A fundamental question in functional Ultrasound imaging remains to date the number of activated neurons required to detect a subtle blood volume change. Here, we give for the first-time preliminary answers to this open question by demonstrating that fUS imaging is sensitive enough to detect functional activations of trigeminal corneal nociceptors known to represent a very small number of neurons (approx. 300).

\section{Imaging the hemodynamic responses in the ipsilateral TG induced by corneal} stimulations: a valuable model to study the trigeminal neurovascular coupling.

This study aimed at providing a proof of concept for the hemodynamic responses in the TG following corneal nociceptive activation. We postulated that corneal stimulations and imaging of the TG in anesthetized animals constitutes a highly interesting model because corneal nociceptors ( $\mathrm{C}$ and A delta corneal fibers) can be easily activated by mechanical stimulations (35) and by chemical stimulation of the TRPV1 receptor by capsaicin (28). 
The ability to detect clear hemodynamic changes in response to corneal stimulations was a particular challenge in this study due to the small number of neurons innervating the cornea.

Despite these challenges, our study demonstrates significant real-time (400 ms) imaging of hemodynamic responses in TG to repeated sequences of $\mathrm{C}$ and A delta fibers mechanical activations. This ipsilateral activation, located in the V1 branch of the TG, is consistent with the known anatomy of the TG in rats (36) and somatotopy of corneal afferents within the TG (31). We went further in our investigations by evaluating the hemodynamic changes after activation of polymodal nociceptors by capsaicin, a TRPV1 agonist. We observed a strong phasic hemodynamic response in the ophthalmic division; the phasic aspect of this response is thought to be related both to difficulty to perform block design experiments, and also to the potent action of capsaicin known to strongly activate and sensitize the sensory afferents $(1,7)$.

\section{The atypical vascularization of the TG}

Until recently (37), the vascularization of the TG has been mostly studied and described in human subjects (38). Here we precisely localized the TG in rats using 2D or 3D fUS imaging and noted a high signal of blood volume,-demonstrating that TG is richly vascularized. In toto staining of the vasculature (DiI experiments) and ULM in vivo, confirmed that rat TG is richly vascularized, with highly tortuous vessels, especially in the cell body rich area (CBRA). Such morphological features are in agreement with the observations of Jimenez-Andrade, et al, who reported a 7-time denser network of vascularization in the CBRA, compared to the nerve fibers rich area (NFRA) within the TG (39). Interestingly, a similar striking regional difference in the expression of tight junction proteins and the presence of functional blood-brain -barrier (BBB) in the NFRA of the 
dorsal root ganglion (DRG), but not the CBRA of the DRG has been reported (40). Indeed, blood vessels that vascularize the CBRA have large fenestrations, when compared to peripheral nerves, see for review $(39,41)$. Some studies exploring the permeability of the BBB showed that injected macromolecules are kept in the blood vessels in the NFRA, while they leak out from the blood vessels in the CBRA (42). Therefore, there is an important dichotomy in the nature of the vascularization in the TG (and DRG) ganglia: while the NFRA has a minor vascularization and is well protected by the BBB, the CBRA on the other hand, has a dense vascularization, with a large lack of BBB. In addition to the high metabolic demand at the level of the cell bodies, these elements are likely the fundamental elements underlying the strong hemodynamic response to orofacial stimulations observed in rats in our study and human subjects.

\section{Mechanisms of neurovascular coupling in the trigeminal ganglion}

Much is still to be understood in the mechanism underlying the neurovascular coupling in the TG and its particular vascularization in health and disease. Trigeminal nociceptive nerve fibers are known to express vasoactive neuropeptides, including CGRP and substance P (43), that are released upon stimulation causing vasodilation that results in perivascular changes, CGRP is considered as the strongest vasodilating neuropeptide in human and also participate in sensitization of the trigemino-vascular system, peripheral sensitization and hyperalgesia (44). CGRP is expressed by $50 \%$ of the corneal neurons, $50 \%$ of these them also co-express TRPV1 (45). Therefore, the stimulation of corneal nociceptors both through mechanical stimulation and through TRPV1 activation by capsaicin application in our experimental design induced the release of CGRP. This release was both local in the TG, through a paracrine mechanism, as previously demonstrated (46) and central (in the trigeminal brainstem sensory complex, (44)). 
Once released, CGRP increases local tone, thus further amplifying CGRP-mediated activation of cell population bearing CGRP receptor such as endothelial, glia and mast cells. Interestingly, a preclinical study showed previously, that while retrogradely traced neurons are localized in clusters in the TG (each branch V1, V2 and V3 being separated), capsaicin induces a spread of the tracer to all branch of the TG in hours following injection in the temporomandibular joint. The tracer was not restricted anymore to neuronal soma, but was detected in satellite cells (46). This phenomenon of cross-excitation within the entire TG induced by capsaicin is thought to be due paracrine neuro-glial communication that leads to communication via gap junctions (46). We propose that these mechanisms, hypothesized previously to be involved in orofacial sensitization and hypersensitivity (46) may also be involved in the increased neurovascular coupling imaged in the TG in our study and in previous clinical studies (4).

In conclusion, this study constitutes the first demonstration of functional mapping of the neurovascular coupling in the rodent TG during corneal stimulations and provides a new experimental approach to investigate the vascular features (blood flow and morphology) and neurovascular coupling under physiological and pathophysiological pain conditions. Future investigations will be useful for the development/the monitoring of therapeutical strategies for debilitating trigeminal chronic pain conditions. 


\section{Figure legends}

Figure 1: Anatomical localization and vascularization of the rat trigeminal ganglia (TG).

A, F: fUS imaging through the whole brain depth reveals the vascularization and localization of the rat TG in a coronal plane (A or sagittal plane: E). B: Capture of the 3D tomographic scan detailed in Supplementary video 1, illustrating the TGs seen in three dimensions (white arrows). C-D: Localization of the trigeminal ganglion according to the Waxholm's tractography atlas (https://scalablebrainatlas.incf.org/rat/PLCJB14, i.e. defined using fiber tracks), observed coronally (C, white arrows) or in 3 Dimensions (D, black surrounding of the ganglia) medio-lateral, at the base of the brain. E-F: In toto vascular staining in the rat TG showing that this structure is richly vascularized by both a dense network of thin (10 $\mu$ m diameter $)$ blood vessels, but also large blood vessels (50 $\mu \mathrm{m}$ diameter). Dil was used to stain the endothelial cell membrane lining the blood vessels, as previously described (47)-IC: Internal carotid. Scale bars $=A, B, C: 2$ mm, E: 1.5mm, F: $200 \mu \mathrm{m}, \mathrm{G}: 100 \mu \mathrm{m}$.

Figure 2: Mechanical and chemical activation of corneal mechano- and polymodal nociceptors induce a functional hyperhaemia in the ipsilateral trigeminal ganglion. A: Schematic presenting the corneal afferents ( $\mathrm{C}$ and A delta fibers) and fUS imaging of the TG. B-C: Double staining CGRP (red) / vascular in toto staining (DiI, Green) showing that the TG is richly vascularized, especially in the 'Cell Body Rich Area', delineated by a dashed white line (B)). Red arrowheads point at CGRP positive sensory neurons located in the near proximity of stained blood vessels (white arrowheads). D-H: Hemodynamic responses in the TG induced by mechanical stimulations of the cornea. D: Example of Doppler image of the brain and trigeminal ganglion, E: map of Z-score and F: delta blood volume (BV) change in a representative example, illustrating the response in the medio-lateral part of the TG. G shows 
the spatially averaged temporal changes in TG's blood flow in the ipsilateral (red) and contralateral (blue) region of interest delineated by the Z-score. H: Quantification of the changes in TG blood volume in 18 trains of stimulations, (obtained from 8 animals) reveals a significant hyperhaemia in the ipsilateral, but not contralateral TG. I-L: Phasic hemodynamic response in the TG induced by activation of the TRPV1 polymodal corneal nociceptors by topical administration of Capsaisin $(10 \mu \mathrm{M})$. I-K: Example of Z-score map (I), delta blood volume (J) and temporal changes of the TG's blood flow (K) in the ipsilateral region of interest delineated by the Z-score in a representative animal. L: quantification of the changes in TG blood volume ( $\mathrm{N}=7$ animals). For all panels, the contralateral area was defined as the symmetric to the ipsilateral ROI. The red crosses in $\mathrm{H}$ and L are outliners. $* \mathrm{P}<0.05$. Scale bars $=$ B: $200 \mu \mathrm{m}, \mathrm{C}: 100 \mu \mathrm{m}, \mathrm{D}, \mathrm{E}, \mathrm{F}, \mathrm{I}, \mathrm{J}: 2 \mathrm{~mm}$.

Figure 3: Ultrasound localization microscopy imaging (ULM) reveals the strong density and high speed of blood flow in the rat trigeminal ganglion in vivo. Following intravenous injection of bio-compatible microbubbles, known as conventional ultrasonic contrast agent (A), allows, the determination of bubble velocity in both the vertical $(B, C, F)$ or horizontal directions (D, G) through the analysis of single bubble trajectories (B-H). Pictures illustrate ULM imaging on a sagittal plane at lateral $=4.2 \mathrm{~mm}$ lateral. A: Doppler image of all bubbles in both the brain and the TG. The TG is surrounded by a white square. B: ULM imaging of the whole brain and TG depicting velocity in the vertical direction. C-D are a higher power magnification of the area delimited in B. C-D-E illustrate the velocity in the vertical (C) and horizontal (D) directions and the field vectors (E) in major blood vessels approaching the TG, which contain two descending veins and one ascending artery. F-G are higher power magnification of $C$ and $D, E$ and $H$ are the field vectors in these blood vessels $€$ and the TG (H). 
These field vectors illustrate the dense vascular network detected in the TG and high speed of blood in these vessels $(9-20 \mathrm{~mm} / \mathrm{sec})$, but also the multiple directions of blood flow within the TG's sub-parts imaged. E, H: The size of the arrows is proportionate to the local speed. Scale bars= A-B: $2 \mathrm{~mm}, \mathrm{C}-\mathrm{D}-\mathrm{E}: 250 \mu \mathrm{m}, \mathrm{F}-\mathrm{G}-\mathrm{H}: 100 \mu \mathrm{m}$. The color bar in G is common for panels B-D and F-G.

Supplementary figure 1: Comparative localization of the trigeminal ganglion imaged using DTI (tractography's atlas from Waxholm, A) and fUS (Ultrafast Doppler, B) at different anteroposterior coordinates in the rat.

Supplementary figure 2: Functional ultrasound imaging of the evoked hyperhaemia in the S1BF induced by whisker pad 's stimulation. A: Ipsilateral stimulation of the right whisker pad known to induce a local hemodynamic response in the contralateral primary sensory cortex, barrel field (S1BF). Failure to observe such a response at the beginning of our experiments induced the exclusion of the animal from the protocol. B: Example of evoked responses, i.e. statistically significant increased positive pixels (significant Z-score) in a representative animal.

Supplementary video 1: Video showing 3-dimension tomographic scan performed in a rat (after removal of the skull to avoid attenuation). It shows the entire vasculature of the rat brain and TGs (below).

Supplementary video 2: Video showing, in real time in a typical experiment (using Iconeus One imager and the software 'Neuroshop'), the increased blood volume in the trigeminal ganglion during the application of repeated corneal mechanical stimulations.

\section{Acknowledgments}


The authors wish to thank the CNRS, Inserm and ESPCI for their financial support.

This work was supported by Sorbonne Université and the Institut National de la Santé et de la Recherche Médicale, ANR, LabEx LIFESENSES (ANR-10-LABX-65) and IHU FOReSIGHT (ANR18-IAHU-01).

In addition, this work was supported by the Chair in Biomedical Imaging of the AXA Research Fund, the ANR 'PINCH' (18-CE37-0005-01) and the European Research Council (ERC) Advanced Grant FUSIMAGINE. Line Rahal was supported by a fellowship from the 'Fondation NRJ'-Institut de France award granted to Mickael Tanter.

Authors would like to thank Fanny Joubert and Michella Khoury-Damaa for their early involvement in the project. Finally, this work was supported by the Inserm ART (Technology Research Accelerator) in "Biomedical Ultrasound".

Several panels of figures were made using Biorender (Biorender.com).

\section{Conflict of interest}

MT and TD are co-founders and shareholders of Iconeus company. MT is co-inventor of several patents in the field of neurofunctional ultrasound and ultrafast ultrasound. MT and TD do not have any other financial conflict of interest, nor any non-financial conflict of interests. All the other authors do not have any financial or non-financial conflict of interests. 


\section{MATERIALS AND METHODS}

\section{Animals}

All experiments were performed in agreement with the European Community Council Directive of September 22, 2010 (010/63/UE) and the local ethics committee (Comité d'éthique en matière d'expérimentation animale number 59,'Paris Centre et Sud', project 2018-05). Accordingly, the number of animals in our study was kept to the necessary minimum. Experiments were performed on N=25 male Sprague-Dawley rats (Janvier Labs; Le Genest St Isle, France), weighing 325-350 g at the beginning of the experiments. Animals (two per cage) arrived in the laboratory 1 week before the beginning of the experiment and were maintained under controlled conditions $\left(22 \pm 1^{\circ} \mathrm{C}, 60 \pm 10 \%\right.$ relative humidity, 12/12h light/dark cycle, food and water ad libitum).

\section{Histological visualization of the blood vessels and Calcitonin Gene related Peptide (CGRP) immunoreactive fibers}

In toto coloration of the trigeminal's vascular architecture

N=3 Sprague-Dawley rats were deeply anesthetized with an intraperitoneal (IP) injection of sodium pentobarbital (Dolethal, Supplier, $150 \mathrm{mg} \cdot \mathrm{kg}^{-1}$ ). Then, a thoracotomy was performed and an incision in the right atrium was made. Animals were perfused with $2 \mathrm{~mL}$ of saline solution $(0.9 \% \mathrm{NaCl})$, followed immediately by $15 \mathrm{~mL}$ of DiI ((Sigma Aldrich, France) 0.012 mg.mL $L^{-1}$ in 2\% ethanol, 98\% PBS (phosphate buffered saline solution, 0.02 mol. $\mathrm{L}^{-1}$ ), as previously described (47)), followed by $10 \mathrm{~mL}$ of paraformaldehyde $4 \%$ at the rate of $7 \mathrm{~mL} \cdot \mathrm{min}^{-1}$. Trigeminal ganglia were extracted and fixed overnight in $4 \%$ paraformaldehyde at $4^{\circ} \mathrm{C}$ before cryoprotection in a $30 \%$ sucrose solution for 1 day. They were frozen in an OCT (Optimal Cutting Temperature) matrix in cooled isopentane ($40^{\circ} \mathrm{C}$ ) on dry ice, cut in $12 \mu \mathrm{m}$ slices on a cryostat (Leica CM $3050 \mathrm{~S}$, Wetzlar, Germany) 
and and mounted on Superfrost slides (Thermofisher scientific, Waltham, Massachusetts, USA). Note: these animals were also used in a previous study on the vasculature of the spinal cord architecture (48).

\section{CGRP immunohistochemistry in the trigeminal ganglion}

After three washes in 0.1 M PBS, TG sections were incubated for $1 \mathrm{~h}$ in a blocking solution of 0.1 M PBS containing 3\% normal donkey serum and $0.1 \%$ triton $\mathrm{X}-100$, followed by incubation with primary antibody at $4^{\circ} \mathrm{C}$ for $24 \mathrm{~h}$. The primary antibody used in this study was mouse anti-CGRP (Sigma-Aldrich: Lot \#083M4785, 1:250). CGRP was revealed using Alexa Fluor 594-conjugated donkey anti-rabbit antibody (1:500; Invitrogen). Finally, the sections were cover slipped.

Microscopic analysis

Tissue sections were examined using a Zeiss M1 epifluorescence microscope (Axio ImagerM1; Carl Zeiss) equipped with a digital camera (C11440-42U30; Hamamatsu Photonics) and an image acquisition software (Zen; Carl Zeiss).

\section{Surgical procedures and preparation for imaging}

Under deep anesthesia (intraperitoneal (IP) bolus of Medetomidine (Domitor, 0,4 mg.kg-

${ }^{-1}$ ) and ketamine (Imalgène, $40 \mathrm{mg} \cdot \mathrm{kg}^{-1}$ )), the animal was placed on a stereotaxic frame and a craniotomy (removal of the skull) was performed as previously described (19) between Bregma and Lambda. Some ELMA cream (AstraZeneca, UK) was placed in the ear bars in order to prevent discharge from nociceptors at the level of the ears. This window allowed the scanning of a large part of the brain, under which the TG is located. During the surgical procedure and the imaging session, the animals' body temperature was kept at $37^{\circ} \mathrm{C}$ using a heating blanket and an intrarectal probe (Physitemp, USA), and the heart 
and respiratory frequencies were monitored continuously (MouseOxPlus, Ugo Basile, Italy). As previously described $(19,48), 45$ min after induction (when the craniotomy was finished), the anesthesia was maintained but reduced, using subcutaneous perfusion of Medetomidine $(0.1 \mathrm{mg} / \mathrm{kg} / \mathrm{h})$ and ketamine $(12.5 \mathrm{mg} / \mathrm{kg} / \mathrm{h})$ using a syringe pump. As previously observed (48), in order to obtain reproducible results, it was preferable to wait in order to reach stable physiological parameters and a reproducible level of anesthesia (respiratory frequency around 80-90 rpm). Each imaging session lasted from 3 to 4 hours.

Two milliliters of saline solution were gently dropped on the brain (the dura mater was kept intact), followed by echographic gel (Dexco Médical, France). The ultrasonic probe was then positioned just above the window using a 4-axis motorized system on which the ultrasound probe was fixed (19).

\section{D and 3D imaging scans of the brain and TG}

In all animals on which we performed fUS experiments, a linear scan was first performed with a few successive antero-posterior 2D scans at $\left(100 \times 100 \times 400 \mu \mathrm{m}^{3}\right)$ resolution in order to reproducibly recognize the imaging plane of interest containing the TG. The spatial extent of the TG was measured and the probe was positioned in the desired plane of imaging (Bregma $+4.56 \mathrm{~mm}$, see supplementary figure 1 ). This plane corresponds to the medio-anterior part of the TG, where the neurons of the branch V1 are expected to be found (36).

Using a prototype ultrasonic ultrafast neuroimager (Iconeus, Paris, France), in $n=2$ animals, a 3D-scan of the entire window (Bregma to Lambda) was performed with a tomographic approach. This approach based on the acquisition of several linear scans 
with different orientations of the probe enables the reconstruction of 3D angiography with isotropic voxel resolution $\left(100 \times 100 \times 100 \mu \mathrm{m}^{3}\right)$ using a linear ultrasonic probe (49). A total of 19 orientations of the probe was used and for each orientation 81 successive 2D scans were acquired with $0.2 \mathrm{~mm}$ step. The isotropic 3D volume reconstructed (IcoStudio, Iconeus, Paris, France) is shown in supplementary video and the figure 2B.

\section{Peripheral stimulations}

Internal positive control : stimulation of the left whisker pad

To verify the appropriate level of anesthesia, we performed a routine test in each experiments, which consisted in measuring of the evoked haemodynamic response in the barrel cortex (S1BF) induced by stimulation of the ipsilateral whisker pad (Supplementary Figure 1), as previously performed in our seminal article (18). A train of 6 stimulations at $4 \mathrm{~Hz}$ lasting $20 \mathrm{sec}$, separated by $20 \mathrm{sec}$, were performed using an Arduino. In the rare cases $(\mathrm{N}=2$ over 25$)$ where this test did not elicit any response after multiple retests in various planes of the barrel cortex, the animal was euthanized and excluded from the experiment.

\section{Mechanical and chemical corneal stimulations}

Mechanical stimulations consisted of 6 manual stimulations of the cornea using a soft brush (gentle stroke, see supplementary video 2). The stimulations lasted $10 \mathrm{sec}$, separated by $20 \mathrm{sec}$, with a resting time of $30 \mathrm{sec}$ before and after these repetitions.

Chemical stimulations were performed using topical corneal application of Capsaisin (10 $\mu \mathrm{M}$, Sigma Aldrich). After $60 \mathrm{sec}$ of baseline (recording in absence of any stimulation), a $2 \mathrm{~mm} X 2 \mathrm{~mm}$ filter paper impregnated with $50 \mu \mathrm{L}$ of $10 \mu \mathrm{M}$ capsaicin was applied on the 
cornea for 1 minute. The cornea was then immediately washed using a saline solution.

\section{fUS imaging: sequences of imaging and signal processing}

fUS imaging was performed using a linear ultrasound probe (128 elements, $15 \mathrm{MHz}, 110$ $\mu \mathrm{m}$ pitch, $8 \mathrm{~mm}$ elevation focus, Iconeus, Paris, France) driven by a prototype ultrasonic ultrafast neuroimager (Iconeus, Paris, France). The fUS imaging sequence operated as follows: the brain and TG were insonified by ten successive tilted plane waves with angles varying from $-10^{\circ}$ to $10^{\circ}$ with a $5.5 \mathrm{kHz}$ Pulse Repetition Frequency (PRF). The backscattered echoes were recorded by the transducer array and beamformed to produce a block of 200 consecutive ultrafast images with a framerate of $500 \mathrm{~Hz}$. In order to filter the Blood Volume (BV) and to remove the tissue signal, we used a clutter filter based on Singular Value Decomposition (SVD) applied to 200 successive frames (50) by removing the 60 first singular vectors which correspond mainly to the tissue space. Finally, a Power Doppler image was obtained by integrating the energy of the filtered frames, resulting in a Power Doppler image every 400ms.

\section{Doppler Signal Analysis and Activation maps}

Doppler data were analyzed using a generalized linear model approach (GLM) implemented in Matlab in order to obtain the Z-score and p-value maps (48). The activation maps show the Z-score of all significant pixels in the image ( $p$-value $<0.05$ (before Bonferroni correction).

We drew the ipsilateral region of interest (ROI) around the activation area thanks to the thresholded Z-score map and the contralateral ROI was drawn by symmetry. The two signals were averaged along the 2 spatial dimensions in order to obtain a single temporal 
signal. The signal was then expressed as a BV (Blood Volume) variation (in percent), or $\Delta \mathrm{BV}$, by subtracting the $\mathrm{BV}$ baseline (calculated for each acquisition by averaging all the temporal data within the TG where the stimulation pattern was strictly equal to 0) and by dividing by the $\mathrm{BV}$ baseline. Mean $\Delta \mathrm{BV}$ values over time were computed at baseline, i.e. during the periods without chemical or mechanical stimulation of the cornea, and during stimulation, i.e. during the periods with stimulation, and are denoted by $\Delta B V_{B L}$ and $\Delta \mathrm{BV}_{\text {STIM }}$

\section{Statistical analysis of the evoked trigeminal hemodynamic responses}

The statistical analysis was performed using Matlab Version 9.7.0.1261785 (R2019b). The data were modelled using linear mixed models (LMM), which are suited to the case of non-independent, hierarchical data $(\Delta \mathrm{BV}$ values from several acquisitions, in several animals), and of factors of interest having fixed effects (ipsilateral vs contralateral TG, baseline vs stimulation) as well as random effect factors (acquisition, animal).

In the case of the corneal stimulation, our aim was to establish the significance of the difference between ipsilateral and contralateral $\Delta \mathrm{BV}$ values during stimulation, with a single stimulation per acquisition, and possibly several acquisitions per rat. The LMM was hence of the form $Y_{i k}=\delta+R_{i}+W_{i k}$, where $Y^{i k}$ denotes the difference between ipsilateral and contralateral $\Delta \mathrm{BV}_{\mathrm{STIM}}$ values for rat i and stimulation $\mathrm{k}, \delta$ denotes the fixed effect of the side, the zero mean $\mathrm{R}_{\mathrm{i}}$ models the random rat effect, and $\mathrm{W}_{\mathrm{ik}}$ is the residual error term. In the case of the mechanical stimulation, our main aim was to establish the significance of the difference between ipsilateral and contralateral $\Delta \mathrm{BV}$ values during stimulation, with 6 stimulations per acquisition, and possibly several acquisitions per rat. The LMM was hence of the form $\mathrm{Y}_{\mathrm{ijk}}=\delta+\mathrm{R}_{\mathrm{i}}+\mathrm{A}_{\mathrm{ij}}+\mathrm{W}_{\mathrm{ijk}}$, where $\mathrm{Y}^{\mathrm{ijk}}$ denotes the difference between ipsilateral and contralateral $\Delta \mathrm{BV}_{\text {STIM }}$ values for rat $\mathrm{i}$, acquisition $\mathrm{j}$, and stimulation $\mathrm{k}, \delta$ 
denotes the fixed effect of the side, the zero mean $R_{\mathrm{i}}$ models the random rat effect, the zero mean $\mathrm{A}_{\mathrm{ij}}$ models the random acquisition effect, and $\mathrm{W}_{\mathrm{ijk}}$ is the residual error term. The same model structure was also used to compare stimulation to baseline $\Delta \mathrm{BV}$ values, either on the ipsilateral $\mathrm{TG}\left(\mathrm{Y}=\Delta \mathrm{BV}_{\mathrm{STI}} \mathrm{M}^{\mathrm{ipsi}}-\Delta \mathrm{BV}_{\mathrm{BL}}{ }^{\mathrm{ipsi}}\right)$, or on the contralateral $\mathrm{TG}(\mathrm{Y}=$

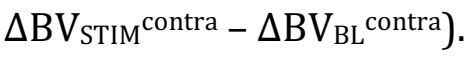

$\Delta \mathrm{BV}$ values were first transformed by taking their square $\operatorname{root}$ (i.e. $\sqrt{ } \Delta \mathrm{BV}$ if $\Delta \mathrm{SB} \geq 0, \sqrt{-} \Delta \mathrm{BV}$ if $\Delta \mathrm{BV}<0$ ) to homogenize their variance and normalize their distributions. The LMMs were fitted with the restricted maximum likelihood method using Matlab's function fitlme. The normality of the residuals was checked with Shapiro-Wilk's test. The test of nullity the fixed effect factor was performed with an F test using Matlab's function fixedEffects. The significance of the random effects was evaluated with a likelihood ratio test using Matlab's function compare.

\section{Ultrasound localization microscopy (ULM)}

Three animals were used for this procedure. A catheter filled with saline was inserted in the rat jugular vein before the positioning of the animal on the stereotaxic frame. ULM was performed similarly to the methods described in (26), but using continuous injections of Sonovue (Braco, Italy) reconstructed in $5 \mathrm{~mL}$ of saline at the rate of $3.5 \mathrm{~mL} / \mathrm{h}$. A total of 750 blocks composed of 400 compounded frames at a $1000 \mathrm{~Hz}$ framerate (with angles at $-5^{\circ},-2^{\circ}, 0^{\circ},+2^{\circ},+5^{\circ}, \mathrm{PRF}=5000 \mathrm{~Hz}, 12 \mathrm{~mm}$ imaging depth) were acquired using the same system as above. The total acquisition lasted 300s. Beamformed data were filtered using the SVD spatio-temporal filter described in (67) and the N=10 first singular values were removed to extract microbubbles signals from the surrounding tissues. Microbubbles were detected as the brightest local maxima in the images. Tracking of the maxima positions was performed using a classical particle tracking algorithm 
(simpletracker.m available on mathworks (C)ean-Yves Tinevez, wrapping matlab munkres algorithm implementation of (CYi Cao 2009). The successive positions gathered in one track were used to compute the interframe bubble velocity vector components (along probe x-axis and depth z-axis) and absolute velocity magnitude. Density maps were computed by counting all the positions detected in one pixel during the acquisitions. Velocity maps were computed as the mean velocity of every MB which passed through one pixel during the whole acquisition. Pixel size for image reconstruction was $\sim 6.5 \mu \mathrm{m}$.

Density maps were computed by counting all the positions detected in one pixel during the acquisitions. Velocity maps are computed as the mean velocity of every microbubble which passed through the pixel during the whole acquisition.

\section{Data availability statement:}

Data supporting the findings associated with the figures of this study will be available after publication on a repository website. 


\section{References}

1. E. A. Moulton, et al., Capsaicin-induced thermal hyperalgesia and sensitization in the human trigeminal nociceptive pathway: An fMRI study. NeuroImage 35, 1586-1600 (2007).

2. D. Borsook, A. F. M. DaSilva, A. Ploghaus, L. Becerra, Specific and Somatotopic Functional Magnetic Resonance Imaging Activation in the Trigeminal Ganglion by Brush and Noxious Heat. J. Neurosci. 23, 7897-7903 (2003).

3. J. Upadhyay, J. Knudsen, J. Anderson, L. Becerra, D. Borsook, Noninvasive mapping of human trigeminal brainstem pathways. Magn. Reson. Med. 60, 1037-1046 (2008).

4. D. Borsook, R. Burstein, L. Becerra, Functional imaging of the human trigeminal system: Opportunities for new insights into pain processing in health and disease. J. Neurobiol. 61, 107-125 (2004).

5. G. Ferrari, et al., Ocular surface injury induces inflammation in the brain: in vivo and ex vivo evidence of a corneal-trigeminal axis. Invest. Ophthalmol. Vis. Sci. 55, 6289-6300 (2014).

6. H. Hirata, K. Mizerska, C. F. Marfurt, M. I. Rosenblatt, Hyperosmolar Tears Induce Functional and Structural Alterations of Corneal Nerves: Electrophysiological and Anatomical Evidence Toward Neurotoxicity. Invest Ophthalmol Vis Sci 56, 8125-8140 (2015).

7. A. Hatta, et al., Dry eye sensitizes cool cells to capsaicin-induced changes in activity via TRPV1. J Neurophysiol 121, 2191-2201 (2019).

8. A. Guerrero-Moreno, C. Baudouin, S. Melik Parsadaniantz, A. Réaux-Le Goazigo, Morphological and Functional Changes of Corneal Nerves and Their Contribution to Peripheral and Central Sensory Abnormalities. Front. Cell. Neurosci. 14 (2020).

9. M. Rothermel, B. S. W. Ng, A. Grabska-Barwińska, H. Hatt, D. Jancke, Nasal Chemosensory-Stimulation Evoked Activity Patterns in the Rat Trigeminal Ganglion Visualized by In Vivo Voltage-Sensitive Dye Imaging. PLOS ONE 6, e26158 (2011).

10. J. E. Hartung, M. S. Gold, GCaMP as an indirect measure of electrical activity in rat trigeminal ganglion neurons. Cell Calcium 89, 102225 (2020).

11. M. Hu, Visualization of Trigeminal Ganglion Neuronal Activities in Mice. Current Protocols in Cell Biology 83, e84 (2019).

12. C. Demene, et al., Functional ultrasound imaging of brain activity in human newborns. Science Translational Medicine 9 (2017).

13. S. Soloukey, et al., Functional Ultrasound (fUS) During Awake Brain Surgery: The Clinical Potential of Intra-Operative Functional and Vascular Brain Mapping. Front. Neurosci. 13, 1384 (2020). 
14. A. Dizeux, et al., Functional ultrasound imaging of the brain reveals propagation of taskrelated brain activity in behaving primates. Nat Commun 10, 1400 (2019).

15. L.-A. Sieu, et al., EEG and functional ultrasound imaging in mobile rats. Nat Methods 12, 831-834 (2015).

16. A. Urban, et al., Real-time imaging of brain activity in freely moving rats using functional ultrasound. Nature Methods 12, 873-878 (2015).

17. A. Bergel, T. Deffieux, C. Demené, M. Tanter, I. Cohen, Local hippocampal fast gamma rhythms precede brain-wide hyperemic patterns during spontaneous rodent REM sleep. Nat Commun 9, 5364 (2018).

18. E. Mace E., et al., Functional ultrasound imaging of the brain. Nat Methods 8, 662-664 (2011).

19. L. Rahal, et al., Ultrafast ultrasound imaging pattern analysis reveals distinctive dynamic brain states and potent sub-network alterations in arthritic animals. Scientific Reports 10, $1-17$ (2020).

20. D. Boido, et al., Mesoscopic and microscopic imaging of sensory responses in the same animal. Nature Communications (2019) https:/doi.org/10.1038/s41467-019-09082-4.

21. C. Iadecola, The Neurovascular Unit Coming of Age: A Journey through Neurovascular Coupling in Health and Disease. Neuron 96, 17-42 (2017).

22. B. F. Osmanski, et al., Functional ultrasound imaging reveals different odor-evoked patterns of vascular activity in the main olfactory bulb and the anterior piriform cortex. NeuroImage 95, 176-184 (2014).

23. É. Macé, et al., Whole-Brain Functional Ultrasound Imaging Reveals Brain Modules for Visuomotor Integration. Neuron 100, 1241-1251.e7 (2018).

24. C. Bimbard, et al., Multi-scale mapping along the auditory hierarchy using highresolution functional UltraSound in the awake ferret. eLife 7, e35028 (2018).

25. C. Errico, et al., Ultrafast ultrasound localization microscopy for deep super-resolution vascular imaging. Nature 527 (2015).

26. C. Demené, et al., Transcranial ultrafast ultrasound localization microscopy of brain vasculature in patients. Nature Biomedical Engineering 5, 219-228 (2021).

27. F. Joubert, et al., Effects of corneal injury on ciliary nerve fibre activity and corneal nociception in mice: A behavioural and electrophysiological study. Eur J Pain 23, 589602 (2019).

28. C. Belmonte, A. Aracil, M. C. Acosta, C. Luna, J. Gallar, Nerves and Sensations from the Eye Surface. The Ocular Surface 2, 248-253 (2004).

29. R. Bakker, P. Tiesinga, R. Kötter, The Scalable Brain Atlas: Instant Web-Based Access to Public Brain Atlases and Related Content. Neuroinform 13, 353-366 (2015). 
30. K. K. Osen, J. Imad, A. E. Wennberg, E. A. Papp, T. B. Leergaard, Waxholm Space atlas of the rat brain auditory system: Three-dimensional delineations based on structural and diffusion tensor magnetic resonance imaging. Neuroimage 199, 38-56 (2019).

31. P.-S. Launay, et al., Combined 3DISCO clearing method, retrograde tracer and ultramicroscopy to map corneal neurons in a whole adult mouse trigeminal ganglion. Exp Eye Res 139, 136-143 (2015).

32. T. Opacic, et al., Motion model ultrasound localization microscopy for preclinical and clinical multiparametric tumor characterization. Nature Communications 9, 1527 (2018).

33. S. K. Piechnik, P. A. Chiarelli, P. Jezzard, Modelling vascular reactivity to investigate the basis of the relationship between cerebral blood volume and flow under $\mathrm{CO} 2$ manipulation. NeuroImage 39, 107-118 (2008).

34. P. J. Goadsby, et al., Pathophysiology of Migraine: A Disorder of Sensory Processing. Physiol Rev 97, 553-622 (2017).

35. M. D. Boada, Relationship between electrophysiological signature and defined sensory modality of trigeminal ganglion neurons in vivo. Journal of Neurophysiology 109, 749757 (2013).

36. J. S. Schneider, F. J. Denaro, U. E. Olazabal, H. O. Leard, Stereotaxic atlas of the trigeminal ganglion in rat, cat, and monkey. Brain Research Bulletin 7, 93-95 (1981).

37. M. Ćetković, et al., Arterial supply of the trigeminal ganglion, a micromorphological study. Folia Morphol. 79, 7 (2020).

38. P. Hendrix, C. J. Griessenauer, P. Foreman, M. M. Shoja, R. S. Tubbs, "Blood Supply of the Cranial Nerves" in Nerves and Nerve Injuries, (Elsevier, 2015), pp. 427-438.

39. J. M. Jimenez-Andrade, et al., Vascularization of the Dorsal Root Ganglia and Peripheral Nerve of the Mouse: Implications for Chemical-Induced Peripheral Sensory Neuropathies. Mol Pain 4, 1744-8069-4-10 (2008).

40. H. Hirakawa, et al., Regional differences in blood-nerve barrier function and tightjunction protein expression within the rat dorsal root ganglion. Neuroreport 15, 405-408 (2004).

41. J. M. Jacobs, Vascular permeability and neurotoxicity. Environ Health Perspect 26, 107$116(1978)$.

42. J. M. Jacobs, R. M. Macfarlane, J. B. Cavanagh, Vascular leakage in the dorsal root ganglia of the rat, studied with horseradish peroxidase. J Neurol Sci 29, 95-107 (1976).

43. K. Messlinger, L. K. Balcziak, A. F. Russo, Cross-talk signaling in the trigeminal ganglion: role of neuropeptides and other mediators. $J$ Neural Transm 127, 431-444 (2020).

44. S. Iyengar, M. H. Ossipov, K. W. Johnson, The role of calcitonin gene-related peptide in peripheral and central pain mechanisms including migraine. Pain 158, 543-559 (2017). 
45. A. Alamri, R. Bron, J. A. Brock, J. J. Ivanusic, Transient receptor potential cation channel subfamily $\mathrm{V}$ member 1 expressing corneal sensory neurons can be subdivided into at least three subpopulations. Front Neuroanat 9, 71 (2015).

46. S. Thalakoti, et al., Neuron-glia signaling in trigeminal ganglion: implications for migraine pathology. Headache 47, 1008-1023; discussion 24-25 (2007).

47. Y. Li, et al., Direct labeling and visualization of blood vessels with lipophilic carbocyanine dye DiI. Nat Protoc 3, 1703-1708 (2008).

48. J. Claron, et al., Large scale functional ultrasound imaging of the spinal cord reveals in depth spatiotemporal responses of spinal nociceptive circuits in both normal and inflammatory state. Pain (2020) https:/doi.org/10.1097/j.pain.0000000000002078.

49. A. Bertolo, et al., Whole-Brain 3D Activation and Functional Connectivity Mapping in Mice using Transcranial Functional Ultrasound Imaging. JoVE (Journal of Visualized Experiments), e62267 (2021).

50. C. Demené, et al., Spatiotemporal Clutter Filtering of Ultrafast Ultrasound Data Highly Increases Doppler and fUltrasound Sensitivity. IEEE Transactions on Medical Imaging 34, 2271-2285 (2015). 


\section{Authors contribution statement}

ARLG, LR and SP designed the experimental paradigm.

LR, ARLG, JC and MTh performed the experiments.

$\mathrm{LR}, \mathrm{BB}, \mathrm{MN}$ and $\mathrm{NR}$ analyzed the data, interpreted the data prepared the figures and wrote some parts of the manuscript.

ARLG and SP wrote the manuscript.

MTa and TD supervised the signal processing, wrote some parts of the manuscript and acquisition/processing software.

IR analysed the statistics, interpreted the data and wrote some parts of the manuscript. NB : ARLG: Annabelle Reaux-Le-Goazigo - MTh: Miguel Thibault - MTa: Mickael Tanter 


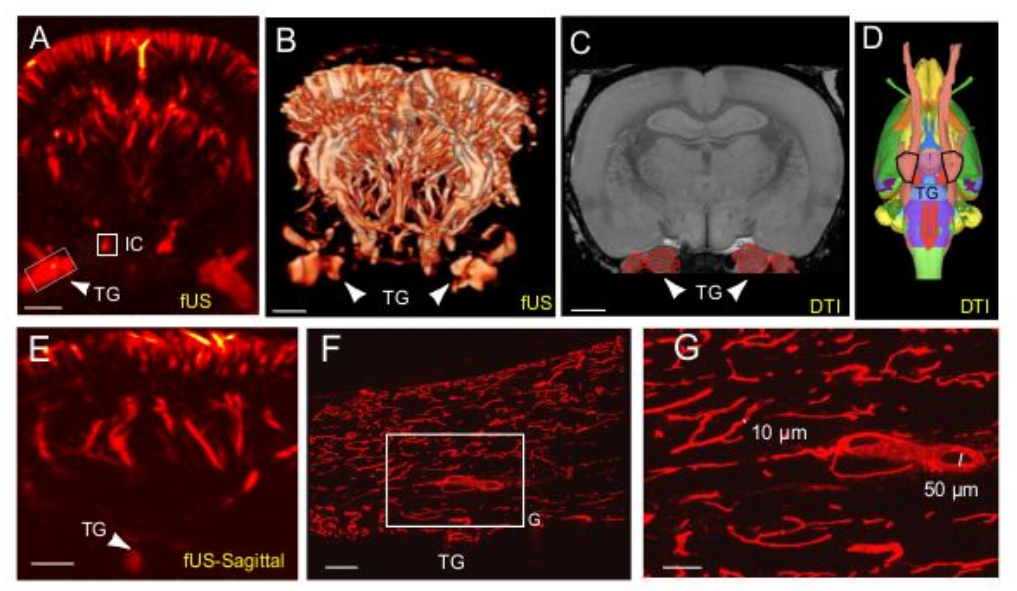

\section{Figure 1}

Anatomical localization and vascularization of the rat trigeminal ganglia (TG). A, F: fUS imaging through the whole brain depth reveals the vascularization and localization of the rat TG in a coronal plane (A or sagittal plane: E). B: Capture of the 3D tomographic scan detailed in Supplementary video 1, illustrating 
the TGs seen in three dimensions (white arrows). C-D: Localization of the trigeminal ganglion according to the Waxholm's tractography atlas (https://scalablebrainatlas.incf.org/rat/PLCJB14, i.e. defined using fiber tracks), observed coronally (C, white arrows) or in 3 Dimensions (D, black surrounding of the ganglia) medio-lateral, at the base of the brain. E-F: In toto vascular staining in the rat TG showing that this structure is richly vascularized by both a dense network of thin (10 $\mu \mathrm{m}$ diameter) blood vessels, but also large blood vessels ( $50 \mu \mathrm{m}$ diameter). Dil was used to stain the endothelial cell membrane lining the blood vessels, as previously described (47). IC: Internal carotid. Scale bars = A, B, C: 2 mm, E: 1.5mm, F: $200 \mu \mathrm{m}, \mathrm{G}: 100 \mu \mathrm{m}$. 

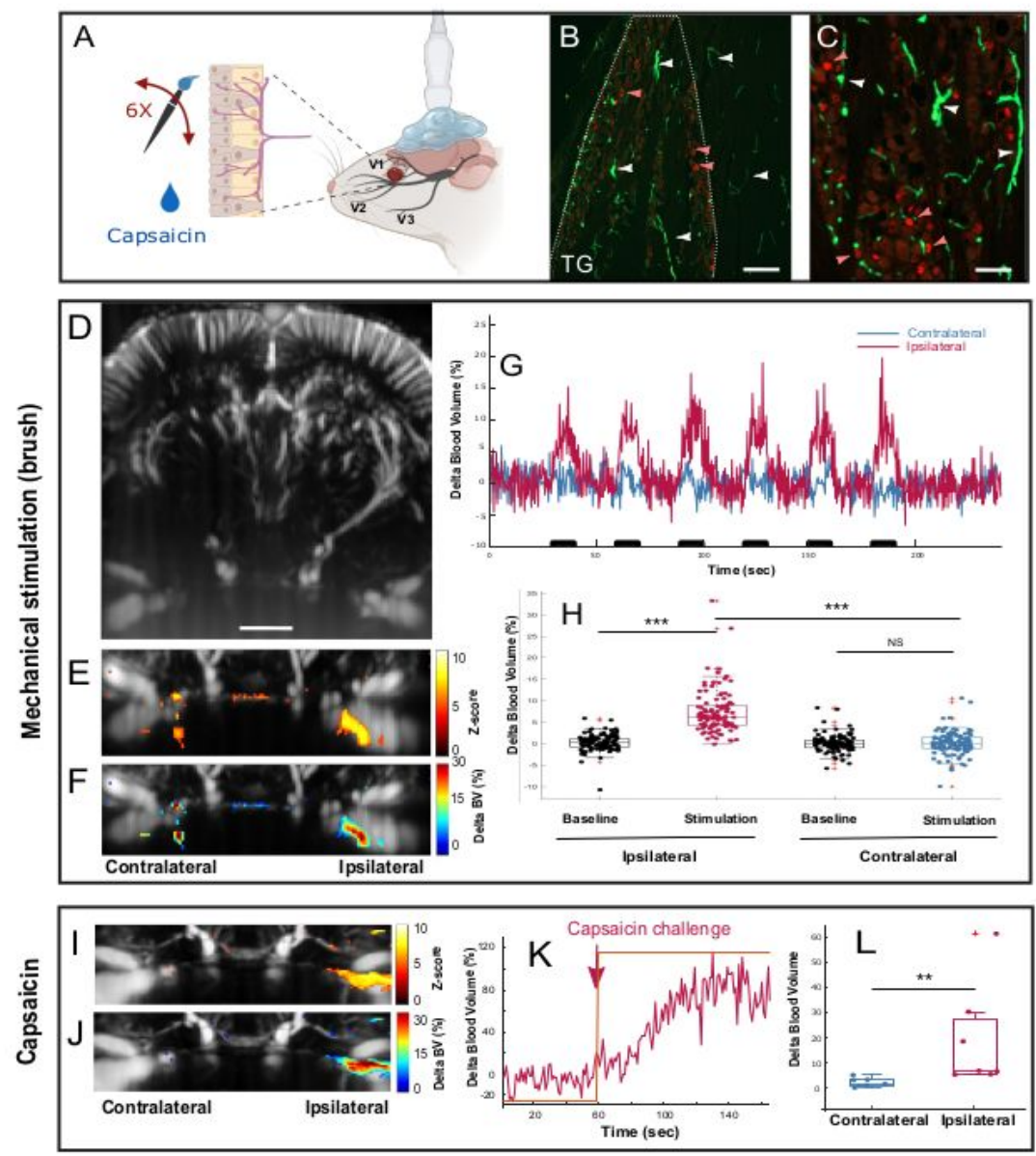

\section{Figure 2}

Mechanical and chemical activation of corneal mechano- and polymodal nociceptors induce a functional hyperhaemia in the ipsilateral trigeminal ganglion. $A$ : Schematic presenting the corneal afferents $(C$ and $A$ delta fibers) and fUS imaging of the TG. B-C: Double staining CGRP (red) / vascular in toto staining (Dil, Green) showing that the TG is richly vascularized, especially in the 'Cell Body Rich Area', delineated by a dashed white line (B)). Red arrowheads point at CGRP positive sensory neurons located in the near 
proximity of stained blood vessels (white arrowheads). D-H: Hemodynamic responses in the TG induced by mechanical stimulations of the cornea. D: Example of Doppler image of the brain and trigeminal ganglion, E: map of Z-score and F: delta blood volume (BV) change in a representative example, illustrating the response in the medio-lateral part of the TG. G shows 13 the spatially averaged temporal changes in TG's blood flow in the ipsilateral (red) and contralateral (blue) region of interest delineated by the Z-score. H: Quantification of the changes in TG blood volume in 18 trains of stimulations, (obtained from 8 animals) reveals a significant hyperhaemia in the ipsilateral, but not contralateral TG. I-L: Phasic hemodynamic response in the TG induced by activation of the TRPV1 polymodal corneal nociceptors by topical administration of Capsaisin $(10 \mu \mathrm{M})$. I-K: Example of Z-score map (I), delta blood volume $(\mathrm{J})$ and temporal changes of the TG's blood flow $(K)$ in the ipsilateral region of interest delineated by the Z-score in a representative animal. L: quantification of the changes in TG blood volume ( $\mathrm{N}=7$ animals). For all panels, the contralateral area was defined as the symmetric to the ipsilateral ROI. The red crosses in $\mathrm{H}$ and $L$ are outliners. ${ }^{*} P<0.05$. Scale bars $=B: 200 \mu \mathrm{m}, C: 100 \mu \mathrm{m}, \mathrm{D}, \mathrm{E}, \mathrm{F}, \mathrm{I}, \mathrm{J}: 2 \mathrm{~mm}$. 

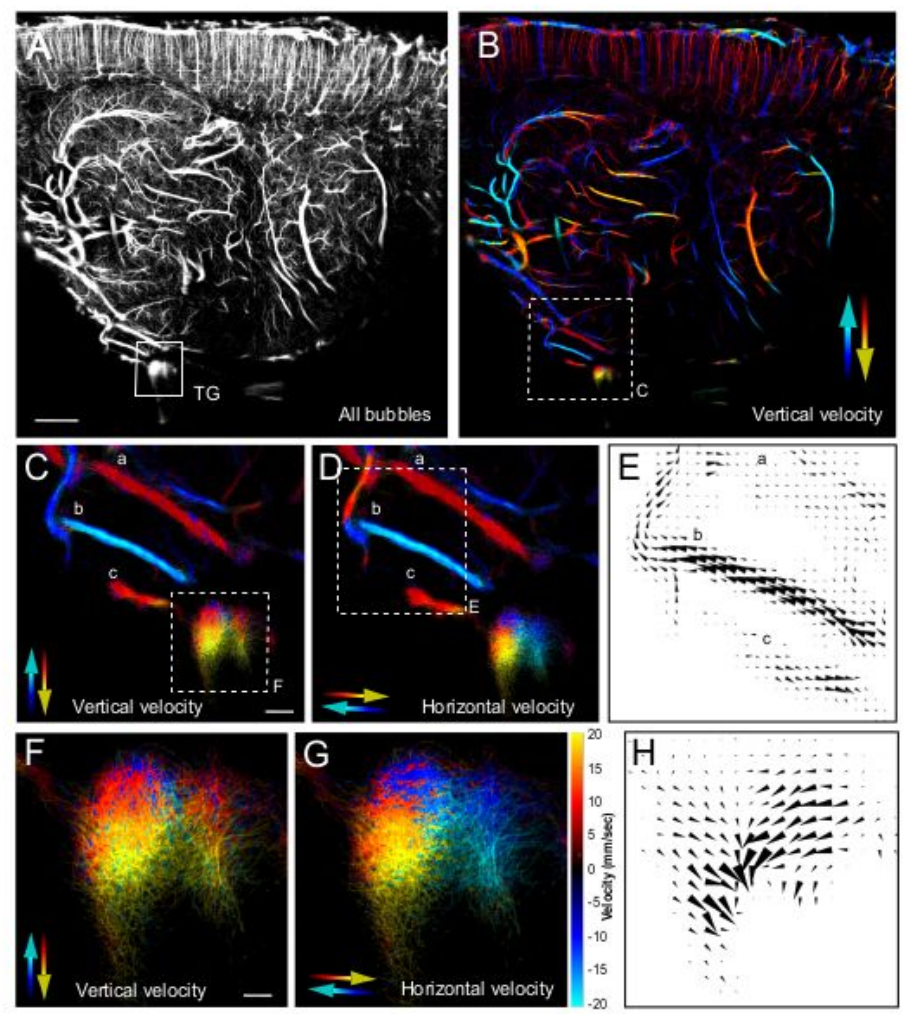

\section{Figure 3}

Ultrasound localization microscopy imaging (ULM) reveals the strong density and high speed of blood flow in the rat trigeminal ganglion in vivo. Following intravenous injection of bio-compatible microbubbles, known as conventional ultrasonic contrast agent $(A)$, allows, the determination of bubble velocity in both the vertical $(B, C, F)$ or horizontal directions $(D, G)$ through the analysis of single bubble trajectories $(B-H)$. Pictures illustrate ULM imaging on a sagittal plane at lateral $=4.2 \mathrm{~mm}$ lateral. $A$ : 
Doppler image of all bubbles in both the brain and the TG. The TG is surrounded by a white square. B: ULM imaging of the whole brain and TG depicting velocity in the vertical direction. C-D are a higher power magnification of the area delimited in B. C-D-E illustrate the velocity in the vertical (C) and horizontal (D) directions and the field vectors (E) in major blood vessels approaching the TG, which contain two descending veins and one ascending artery. F-G are higher power magnification of $C$ and $D, E$ and $H$ are the field vectors in these blood vessels $€$ and the TG $(H)$. 14 These field vectors illustrate the dense vascular network detected in the TG and high speed of blood in these vessels $(9-20 \mathrm{~mm} / \mathrm{sec})$, but also the multiple directions of blood flow within the TG's sub-parts imaged. $\mathrm{E}, \mathrm{H}$ : The size of the arrows is proportionate to the local speed. Scale bars= A-B: 2 mm, C-D-E: $250 \mu \mathrm{m}, \mathrm{F}-\mathrm{G}-\mathrm{H}: 100 \mu \mathrm{m}$. The color bar in G is common for panels B-D and F-G.

\section{Supplementary Files}

This is a list of supplementary files associated with this preprint. Click to download.

- Suplemfigure1.pdf

- Suplemfigure2.pdf

- Supplementaryvideo1.mp4

- Supplementaryvideo24MB.mp4 